\title{
Preparation of Dry Dispersible Emulsion (DDE) to Enhance The Dissolution Rate of Curcumin
}

\author{
Mahdi Jufri ${ }^{\star}$, Miftahul Huda, Abdul Munim \\ Faculty of Pharmacy, Universitas Indonesia \\ Depok 16424, Indonesia
}

\begin{abstract}
Curcumin is an active ingredient obtained from the turmeric plant and has been reported to have many biological activities as anti cancer, an anti-inflammatory, antimicrobial and antioxidant although its clinical use is limited because of its poor solubility in water and inadequate dissolution. Objective- The aim of this research is to prepare dry dispersible emulsion (DDE) of curcumin and to know its effect on enhancing the dissolution rate of curcumin. Method-The dry dispersible emulsion was prepared byusing a high-speed homogenization and ultrasonic technique. Caseinate sodium was used as the surfactant while virgin coconut oil was used as the lipid. Dispersion of the dry emulsion was then spray dried. Dry dispersible emulsion powder was characterized and compared with standard curcumin. Result-The DSC test showed a significant decrease in the melting point. Conclusion-The dissolution rate of curcumin can be significantly improved with a dry dispersible emulsion formulation. In formula $\mathrm{A}$ and formula $\mathrm{C}$, the maximum dissolved curcumin increased by $83.65 \%, 81.53 \%$ in formula $\mathrm{B}$, and $79.12 \%$ in formula $\mathrm{C}$.
\end{abstract}

Keywords: curcumin, drug dispersible emulsion, spray dried, dissolution.

\section{Introduction}

Dissolution rate is a factor that limits the absorption of medicines having low solubility in water. Low solubility leads to low bioavailability and very often leads to high variability in absorption kinetics after oral administration ${ }^{1}$. Drugs with low solubility have inefficient clinical usefulness as they do not penetrate well to the body ${ }^{2}$. Curcumin is the active ingredient derived from turmeric. Curcumin has been reported to respond to many biological conditions, including functioning as anti cancer, an anti-inflammatory, antimicrobial and antioxidant ${ }^{3}$, but ineffective in oral absorption due to its poor solubility in water $(11 \mathrm{ng} / \mathrm{ml})$ and its inadequacy in dissolution leads to a low systemic oral bioavailability, limiting the clinical use ${ }^{4}$. Dry dispersible emulsion (DDE) carrier system is an alternative to the usual colloidal carriers such as emulsions, liposomes, and polymeric microparticles. The novelty of this dry emulsions offer the advantages of improved physicochemical stability, ease of manufacturing, improved storage convenience and increased precision of dosing from solid dosage forms as compared to conventional liquid emulsions ${ }^{5,6}$. Many formulation

Mahdi Jufri et al /International Journal of PharmTech Research, 2019,12(2): 162-170. 
approaches have been attempted to improve the dissolution properties of curcumin for faster onset of action and improved bioavailability. Examples include $\mathrm{SLN}^{7}$, solid dispersion ${ }^{8}$, and nanoemulsion ${ }^{9}$. The aim of this study is to create dry dispersible emulsion containing curcumin and determine its effect on increasing the dissolution rate of curcumin.

\section{Materials and Methods}

\section{Materials}

Turmeric extract (curcumin 70\%) (Insular Multi Natural, Indonesia), curcumin (Merck, Germany), maltodextrin (Qinhuangdao Lihua Starch, China), virgin coconut oil (Vermin International, Indonesia), sodium caseinate (Sigma-Aldrich, USA), butyl hydroxy toluene (Brataco, Indonesia), ethanol (Merck, Germany), phosphate buffer pH 6.8 and distilled water. Spray dryer (Buchi mini spray dryer B - 290, Switzerland), homogenizer (VirTis, United States), ultrasonic (Branson 3200, USA), Scanning Electron Microscopy JSM - 6510 (JEOL, UK ), X - ray diffractometer (Shimadzu XRD -7000, Japan), Differential Scanning Calorimetry (DSC - 60A Shimadzu, Japan), Particle Size Analyzer (Malvern, UK ), Zetasizer (Malvern, UK), UV - Vis spectrophotometer (Shimadzu UV -1800, Japan), dissolution tester (Electrolab TDT - 08L, India), analytic balance (EB - 30 Shimadzu, Japan).

\section{Preparation of dry dispersible emulsion}

Maltodextrin was dissolved in distilled water, then sodium caseinate was added, and stirred until it dissolved. This was used as the water phase.Virgin coconut oil (VCO) was used as the lipid phase. Curcumin was the model drug and Butyl Hydroxy Toluene (BHT) was the antioxidant dispersed into the VCO (Table 1 is the composition of each formula).In the second phase, the formulas heated above $70^{\circ} \mathrm{C}$ in a water bath then homogenized at 30,000 rpm for 15 minutes using a homogenizer to form a homogeneous DDE. Dry dispersion emulsion is then ultrasonicated for 30 minutes. Spray drying dried the results of dry dispersible emulsion with an inlet temperature of $180^{\circ} \mathrm{C}$ and the outlet temperature of $90^{\circ} \mathrm{C}$. The results of the dry dispersible emulsion powder were stored in a closed container protected from light at room temperature.

\section{Table 1: Composition of each formula DDE}

\begin{tabular}{llll}
\hline DDE composition & Formula A $(\%)$ & Formula B $(\%)$ & Formula C (\%) \\
\hline Curcumin & 1 & 1 & 1 \\
\hline Maltodextrin & 10 & 10 & 10 \\
\hline Sodium caseinate & 7 & 8 & 9 \\
\hline Virgin coconut oil & 10 & 10 & 10 \\
\hline Buttylhydroxytoluene & 0.1 & 0.1 & 0.1 \\
\hline Distilled water & 71.9 & 70.9 & 69.9 \\
\hline
\end{tabular}

\section{Size and particle size distribution}

A particle size analyzer (PSA) was used to determine the size of the dry dispersible emulsion preparations and measure the distribution of particles. DDE powder was dispersed in distilled water, and then inserted into the sample tube. The particle size analyzer was then activated.

\section{Zeta potential measurement}

Small aliquots (fractions) $(100 \mu \mathrm{L})$ ofDDE powders were dispersed in $50.0 \mathrm{~mL}$ of distilled water and immediately measured with a Malvern Zetasizer (UK). 


\section{Morphology of particles}

The shape and morphology of dry dispersible emulsion were observed by using a scanning electron microscopy (SEM) to examine the microscopic structure of the powder particles and the dry dispersible emulsion of standard curcumin powder. Powder sample holders were attached to coated conductor tape; then samples were coated using gold $\mathrm{(Au})$ in a vacuum evaporator apparatus.

\section{Differential Scanning Calorimetry (DSC)}

Tests were carried out on standard curcumin and curcumin-contained DDE powders using Differential Scanning Calorimetry instruments. DSC was used to determine the thermal properties. Some samples (3-5mg) were put into a crucible $40 \mu \mathrm{l}$, and analysis was conducted at temperatures of $30-220^{\circ} \mathrm{C}$, with a temperature rise of $10^{\circ} \mathrm{C} / \mathrm{min}^{8}$.

\section{Entrapment efficiency}

A total of $10.0 \mathrm{mg}$ of DDE containing Curcumin was dissolved in $10 \mathrm{ml}$ of methanol, then centrifuged at $3500 \mathrm{rpm}$ for 30 minutes. Following that, the amount of curcumin absorption in the supernatant was determined at a predetermined wavelengths using a UV - Vis spectrophotometer. The result was then used to calculate the levels of free curcumin using the linear regression equation obtained from the calibration curve?

Entrapment efficiency $(\%)=$ (initial levels of drug - free drug concentration)

(initial concentration of drug) x $100 \%$.

\section{Determination of curcumin in dry dispersible emulsion powder}

This test was performed to determine the amount of curcumin in DDE powders in each formula for the dissolution test. A measure of DDE powder $(50.0 \mathrm{mg}$ ) from each of the formulas was put in a $100.0 \mathrm{~mL}$ volumetric flask, then diluted with a solvent $(60 \% \mathrm{v} / \mathrm{v}$ phosphate buffer and $40 \% \mathrm{v} / \mathrm{v}$ ethanol). The levels of the drug content were then measured using a sphectrophotometer uv- vis at a specify wavelength. The result was then used to calculate the degree of curcumin in each formula using the linear regression equation obtained from the calibration curve.

\section{In vitro dissolution}

The dissolution test was conducted to determine the dissolution rate ratio between standard curcumin with DDE powder containing curcumin, using the dissolution apparatus type I (basket) at $100 \mathrm{rpm} .15 .0 \mathrm{mg}$ curcumin powder was used as the norm, and an equivalent amount of DDE was weighed to be used. Then the powder was wrapped in filter paper before being inserted into the basket. The dissolution medium used was $900 \mathrm{ml}$ phosphate buffer $\mathrm{pH} 6.8$ with a temperature of $37 \pm 0.5^{\circ} \mathrm{C} .10 .0 \mathrm{ml}$ samples were taken at minute 15 , $30,45,60,90,120,150,180,240$, and filtered through a membrane filter $(0.45 \mu \mathrm{m})$. After that, the samples were measured by using a UV - Vis spectrophotometer at the maximum wavelength of the curcumin. Then, the amount of drug in the liquid and the percentage of drug released were calculated to make dissolution profiles. Each time a sample of the medium volume was taken it was replaced with a new medium solution with the same volume and temperature?.

\section{Results and Discussion}

Dry Dispersiible Emulsion (DDE) as a formulation has often been suggested to prevent instability of conventional emulsions arising out of creaming, flocculation, coalescence, and phase separation. Also, DDE has been widely used as an active carrier to formulate poorly soluble or unstable drugs (e.g., light- sensitive or rapidly oxidized compounds ${ }^{10}$. Preparation of dry dispersible emulsion (DDE) was carried out by combining high- speed homogenization techniques and ultrasonics, at a temperature of $70{ }^{\circ} \mathrm{C}$ to get DDE preparations that are homogeneous and have a small particle size. The greatest advantage of these techniques is that the equipment used is simple and commonly found in every laboratory ${ }^{11}$. At the beginning of the study, preliminary experiments were conducted to determine the experimental conditions and the composition of the 
best materials to produce stable and homogeneous preparations of dry dispersible emulsion powder. The dry dispersible emulsionto be formed is the oil in water type. Oil is the internal phase and water is the external phase. In the manufacturing process, the hydrophobic materials are dissolved in the oil phase, while the hydrophilic materials are dissolved in the water phase. The stirring speed used was 30,000 rpm for 15 minutes to reduce the particle size, because at the stirring speed of $20,000 \mathrm{rpm}$ the particles were still micrometer size. Extended periods of agitation also affect the formation of the dry dispersible emulsion. After stirring for 10 minutes, the dry dispersible emulsion had not yet formed, and the ingredients were still not homogeneous. After agitation was increased to 15 minutes, yellow-orange and homogeneous dry dispersible emulsion powder formed. Once the dry dispersible emulsion formed, then underwent ultrasonication for 30 minutes because it was found that the use of ultrasonication for 45 minutes resulted in a too small particle size, i.e., $6.855 \mathrm{~nm}$. However, after only 15 minutes of ultrasonication, the particles were still micrometer-sized.In this study, sodium caseinate was used as an emulsifier. Sodium caseinate is a milk protein compound and is reported to have good thermal stability $\left(\sim 140^{\circ} \mathrm{C}\right)$. The presence of hydrophilic and lipophilic groups in the same polymer chain facilitate protein associated with oil and water, which causes the emulsion to be stable ${ }^{\mathbf{1 2}}$. The use of sodium caseinate concentration varied from $7 \%-9 \%$. This is because the use of sodium caseinate below $7 \%$ caused dry dispersible emulsion to separate into two layers. This separation occurs because the emulsifier concentration used is not sufficient to preclude the merger of the oil droplets. However, if as much as $7 \%, 8 \%$, and $9 \%$ of sodium caseinate were used the dry dispersible emulsion formed was stable, because the concentrations used were enough to form a protective layer to prevent the incorporation of the oil phase droplets. The use of as much as $10 \%$ of sodium caseinate resulted in stable dry dispersible emulsion powder , but the emulsion was very viscous and not suitable for spray drying method.In this experiment virgin coconut oil (VCO) was used in the oil phase at a concentration of $10 \%$, this oil is relatively resistant to heat and has a practical value in health. Also, $10 \%$ of maltodextrin was also used as a cosurfactant to increase the solubility of curcumin in water. In this study, the concentrations of the VCO and maltodextrin are not varied.This formulation also used butyl hydroxy toluene (BHT) as an antioxidant. The degradation of curcumin is known to be inhibited in the presence of antioxidants ${ }^{13}$. The antioxidant activity of curcumin, desmethoxycurcumin, and bisdemethoxycurcumin has been studied by using the thiocyanate method using BHT as a comparison. Compared with BHT, Curcumin, desmethoxycurcumin, and bisdemethoxycurcumin can prevent the oxidation of linoleic acid, respectively at $81.98 \%, 81.77 \%$, and $73 \%$. This suggests that BHT is more easily oxidized than the three curcuminoids ${ }^{14}$. The dry dispersible emulsion was made into three formulas, namely formulas A, B, and C. Drying of liquid DDE was performed by spray drying at $180^{\circ} \mathrm{C}$ inlet temperature and $90^{\circ} \mathrm{C}$ outlet temperature. Spraying speed was at $40 \mathrm{ml} / \mathrm{min}$ and at 4 bar pressure. Dry dispersible emulsion produced from curcumin was in the form of fine yellow colored powder, slightly hygroscopic due to the hygroscopic properties of maltodextrins and having a coconut smell caused by the VCO used as-as the lipid.

\section{Size and particle size Distribution}

The particle size of dry dispersible emulsion powder were measured with particle size analyzer using medium of water. The results of DDE measurements can be seen in Table 2. The particle size of dry dispersible emulsion(DDE) are influenced by the results of spray dried scattering parameters (nozzle size and temperature), but it is also affected by the concentration of lipid and emulsifier solution to be spray dried ${ }^{15}$. Nozzle size used in this drying is $2 \mathrm{~mm}$, whereas the drying temperature is $180^{\circ} \mathrm{C}$ inlet temperature and $90^{\circ} \mathrm{C}$ outlet temperature. Based on the a table 2 it can be seen that DDE formula $\mathrm{C}$ has a larger size than DDE formula $\mathrm{B}$, and DDE formula $\mathrm{B}$ has a larger size than DDE formula A. Because the concentration of emulsifier in formula $\mathrm{C}$ was bigger, the solution viscosity is more important than in methods $\mathrm{B}$ and $\mathrm{A}$. The greater concentration of emulsifier caused larger particle sizes ${ }^{15}$. Likewise, is the lipid concentration, but in this study, the lipid concentration is not varied.

Table 2: Particle size of DDE in each formula

\begin{tabular}{lll}
\hline Formula & Particle size (nm) & Volume $(\%)$ \\
\hline $\mathrm{A}$ & 690.4 & 100 \\
\hline $\mathrm{B}$ & 941.3 & 100 \\
\hline $\mathrm{C}$ & 1151 & 100 \\
\hline
\end{tabular}




\section{Zeta potential measurement}

Zeta potential was measured using a Zetasizer. Zeta potential has many applications in stabilizing the dispersed particles system; this potential was depicted as a degree of repulsion between the dispersed charged particulate matter and resulted particles which close to each other ${ }^{16}$. The test results of the zeta potential of the dry dispersible emulsion in formula $\mathrm{A}$ was $-29.77 \mathrm{mV}$, formula $\mathrm{B}$ was $-21.79 \mathrm{mV}$, and formula $\mathrm{C}$ was $27.51 \mathrm{mV}$. Dry dispersible emulsion particle with a zeta potential above $+/-30 \mathrm{mV}$ has demonstrated a surface charge stability that prevents aggregation of the particles. Zeta potential is also related to physical stability. Lowering the zeta potential can lead to aggregation or sedimentation. Zeta potential of a dry dispersible emulsion is typically used to characterize the properties of the particle's surface charge relating to electrostatic interactions of a dry dispersible emulsion. Zeta potential charge reflects the potential of particulate matter and is influenced by the composition of the particles and the medium of the dispersed nanoparticles ${ }^{17}$. The zeta potential results obtained indicate a relatively stable trait for DDE in formula A and formula $\mathrm{C}$, while formula $\mathrm{B}$ shows that the results are a little unstable. Charge particles showed a negative zeta potential. Most of the particles are dispersed in water to get a negative charge because it tends to absorb hydroxyl ions ${ }^{16}$. The negative charge is also affected by sodium caseinate which is an anionic surfactant.

\section{Morphology of DDE}

This observation was conducted to compare the shape and morphology of standard curcumin with DDE containing curcumin. Shape and morphology observations were performed using a Scanning Electron Microscopy (SEM). These observations were made with 2,000 times magnification. SEM results of regular curcumin powder and DDE powder can be seen in Figure 1. SEM results showed that standard curcumin has a crystalline form that resembles broken needles of different sizes. This form is not visible in the SEM results of DDE powders which have an irregular spherical shape and smooth surface with small holes. The SEM results showed that curcumin is absorbed in DDE powder.

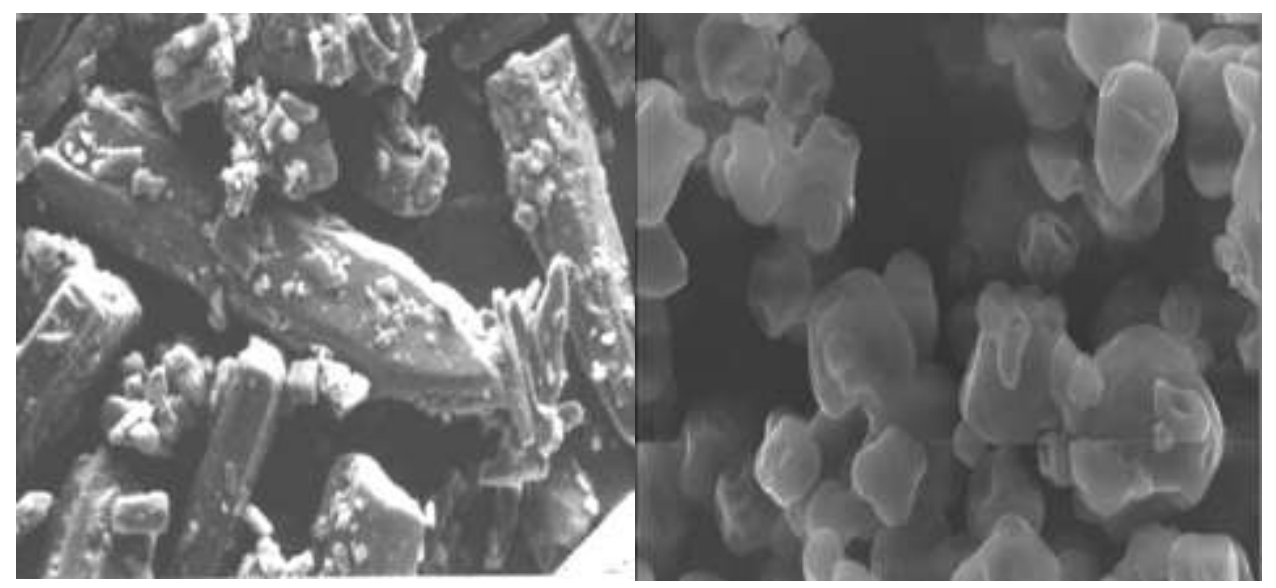

(a)

(b)

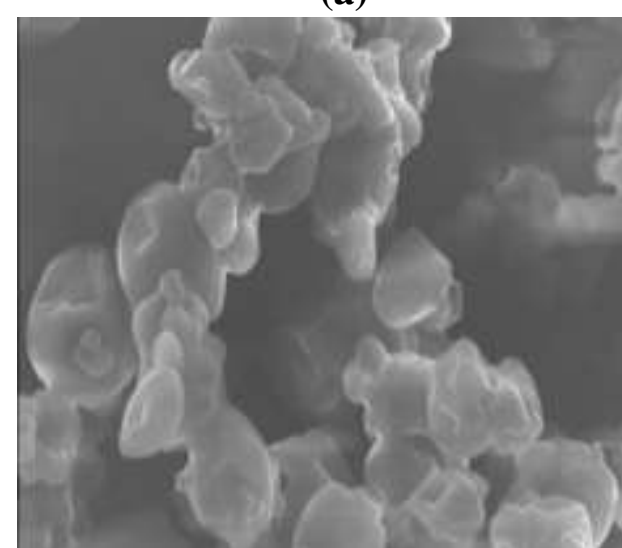

(c)

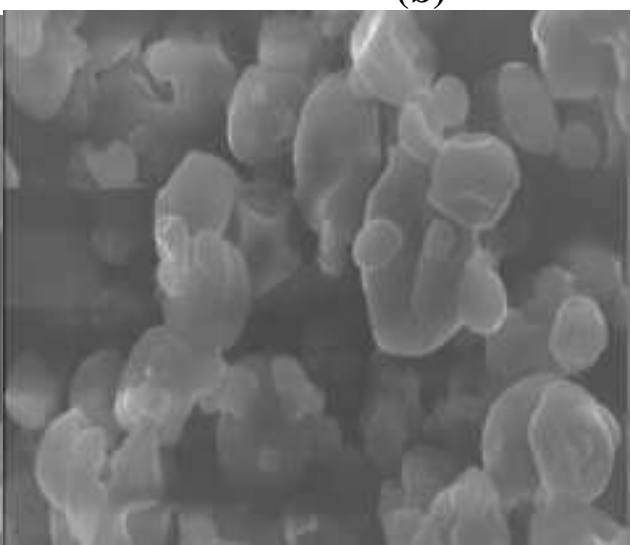

(d)

Fig. 1: SEM results of (a) standard curcumin powder, (b) dry dispersible emulsion powder formula $A$, (c) formula $B$, (d) formula $C$ magnification of $2,000 x$ 


\section{Differential Scanning Calorimetry (DSC)}

The results of thermal analysis using DSC carried out at temperatures of $30-220^{\circ} \mathrm{C}$ with a heating rate of $10^{\circ} \mathrm{C} / \mathrm{min}$ showed a shift in the endothermic peak temperature and enthalpy of standard curcumin. According to the obtained thermogram results, the melting point temperature of standard curcumin and dry dispersible emulsion decreased. The melting point of standard curcumin was $176.74^{\circ} \mathrm{C}$, while for the solid lipid nanoparticles was $147.87^{\circ} \mathrm{C}$ (Figure 2). These thermograms show that the formation of the dry dispersible emulsion was characterized by a change in the crystal structure of the formulations which indicates a significant shift in the melting point. The thermograms showed a decrease in the enthalpy of solid lipid nanoparticles over standard curcumin. The enthalpy $(\Delta \mathrm{H})$ of standard curcumin is $65.70 \mathrm{~kJ} / \mathrm{kg}$, while the $\Delta \mathrm{H}$ of the dry disoersible emulsion powder sample is $62.42 \mathrm{~kJ} / \mathrm{kg}$. The energy required to melt the sample is smaller than the energy required to meet standard curcumin.

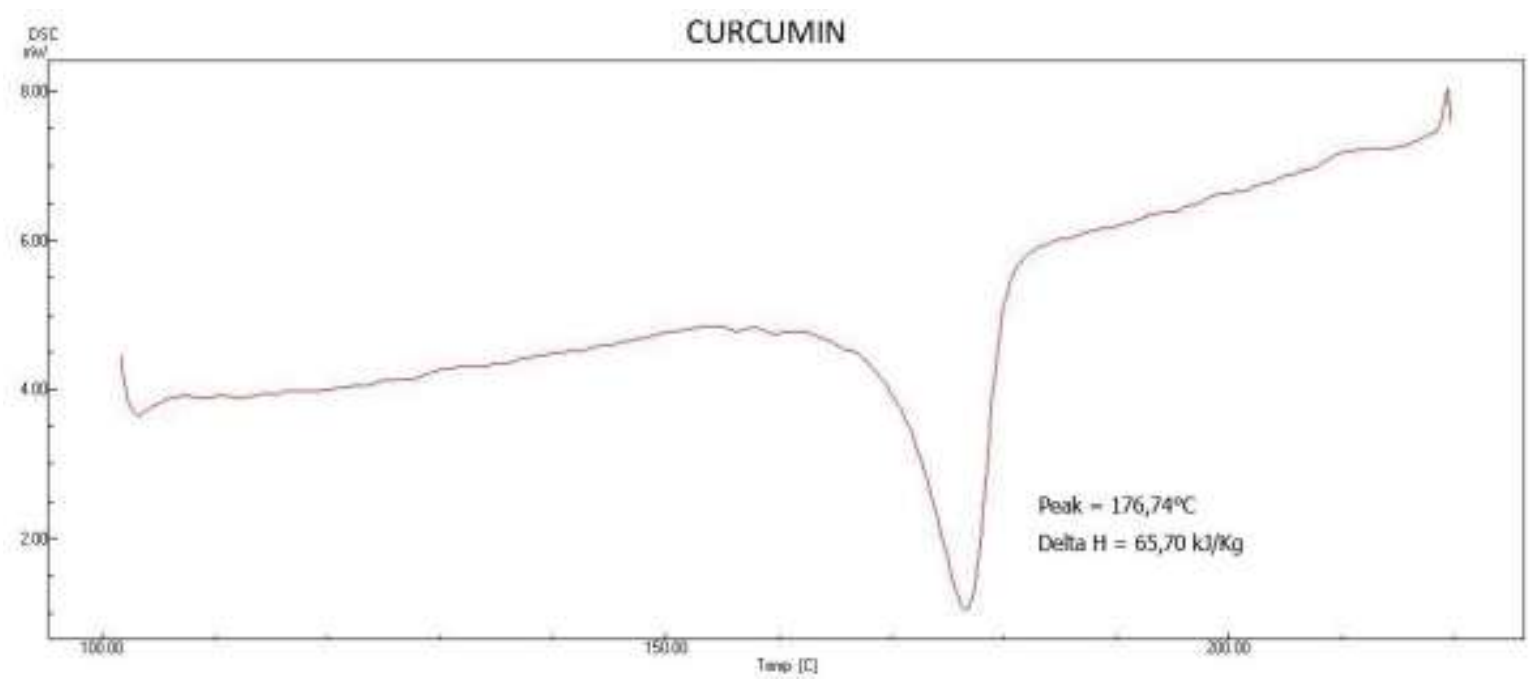

(a)

DDE Curcumin

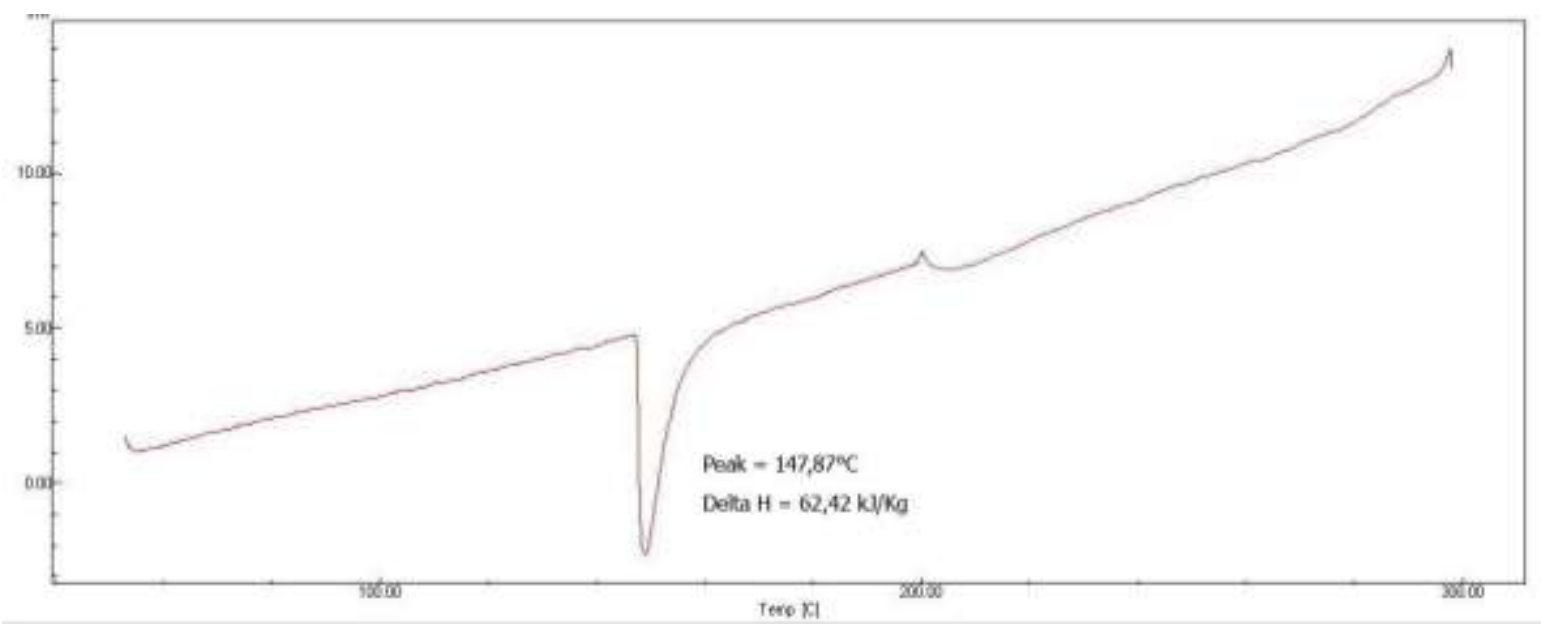

(b)

Fig.2: DSC thermogram of standard curcumin (a) and DDE Curcumin (b)

\section{Entrapment Efficiency}

Entrapment efficiency tests were conducted to determine the adsorbed amount of curcumin in dry dispersible emulsion (DDE)). Based on the adsorption test results, curcumin is adsorbed in DDE formula A at $43.54 \%$, formula B at $48.71 \%$ and formula $\mathrm{C}$ at $52.02 \%$. 


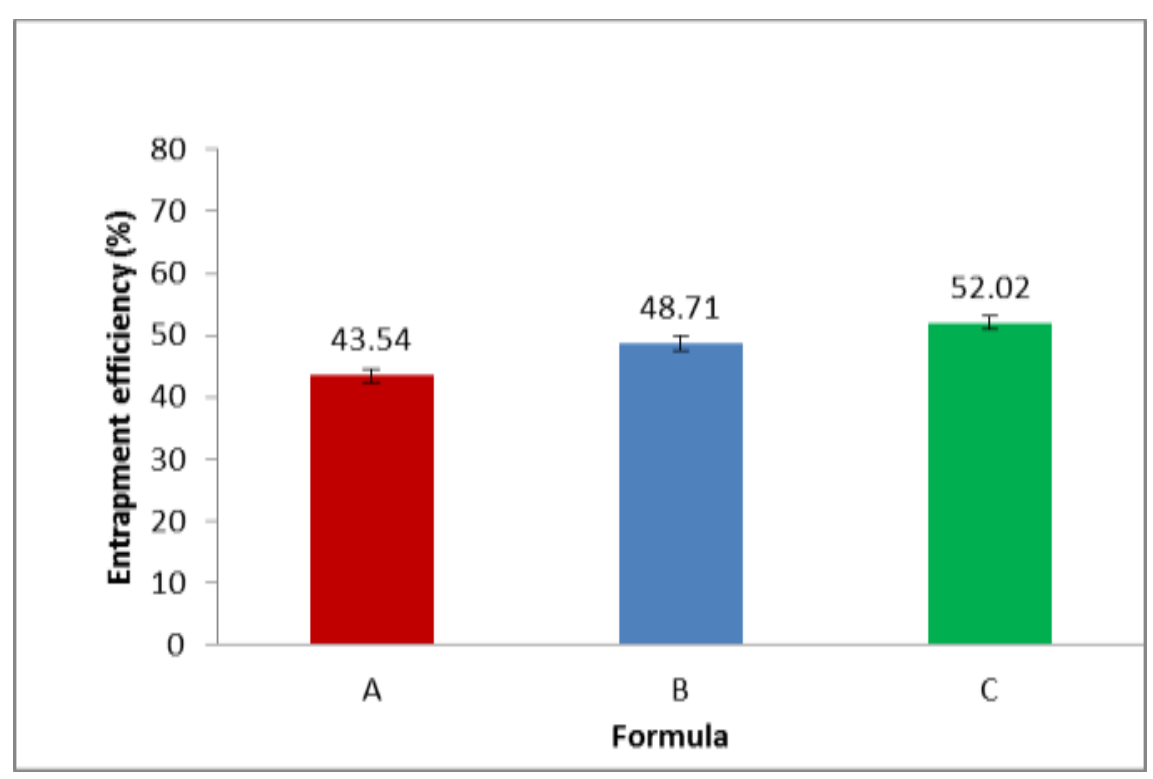

Fig. 3. Entrapment efficiency of DDE

The small amount of curcumin in the DDE adsorption efficiency is due to the low solubility of curcumin in the lipid phase. The factor affecting the adsorption of the drug in DDE is the lipid solubility of the drug in liquids ${ }^{18}$.

The high and low entrapment efficiency may be caused by the addition of a surfactant in the manufacture of DDE. The effect on the amount of surfactant on entrapment efficiency has been tested with various amounts while some surfactant lipids remained constant. The results showed that the adsorption energy accelerated with an increased amount of surfactant. Curcumin in the surfactant layer adsorbed in the DDE led to high entrapment efficiency. Conversely, when amounts of lipid concentration were varied the surfactant entrapment efficiency results still indicated a decrease in an increasing amount of lipids ${ }^{\mathbf{1 9}}$.

\section{Determination of curcumin DDE in the powder}

A curcumin assay test was performed to determine the amount of the drug contained in the dry dispersible emulsion powder from each formula. The resulting levels of curcumin in formula A were $2.03 \%$, Formula B $2.33 \%$, and in formula C $2.48 \%$. High or low levels of curcumin are proportional to the adsorption test results; the results showed that the higher the amount of surfactant the higher is the level of curcumin. The test results of the assay will be used to calculate the number of samples in each formula to be employed in the in vitro dissolution test, so that the amount of curcumin weighed is the same.

\section{In vitro dissolution testing}

The dissolution test performed on DDE powder formulas A, B and C compared to standard curcumin particles using dissolution test apparatus type I (basket) with $\mathrm{pH} 6.8$ phosphate buffer medium at a speed of $100 \mathrm{rpm}$ at a temperature of $37 \pm 0.5^{\circ} \mathrm{C}$. The cumulative dissolution profile of curcumin can be seen in Figure 4. When DDE powder comes contact with water it will form emulsion and it will effect release of curcumin. The results of the dissolution test show that formula A the maximum dissolved curcumin increased by $83.65 \%$ at 120 minutes, $81.53 \%$ of formula $\mathrm{B}$ at 120 minutes, and $79.12 \%$ of formula $\mathrm{C}$ at 120 minutes, while the maximum dissolution of regular curcumin powder increased by $18.83 \%$ at 150 minutes. Even though formula $\mathrm{C}$ has more sodium caseinate and more drug entrapment but still show less release rate because sodium caseinate is a surfactant which its critical micelle concentration was achieved and the surface tension did not decrease again. 


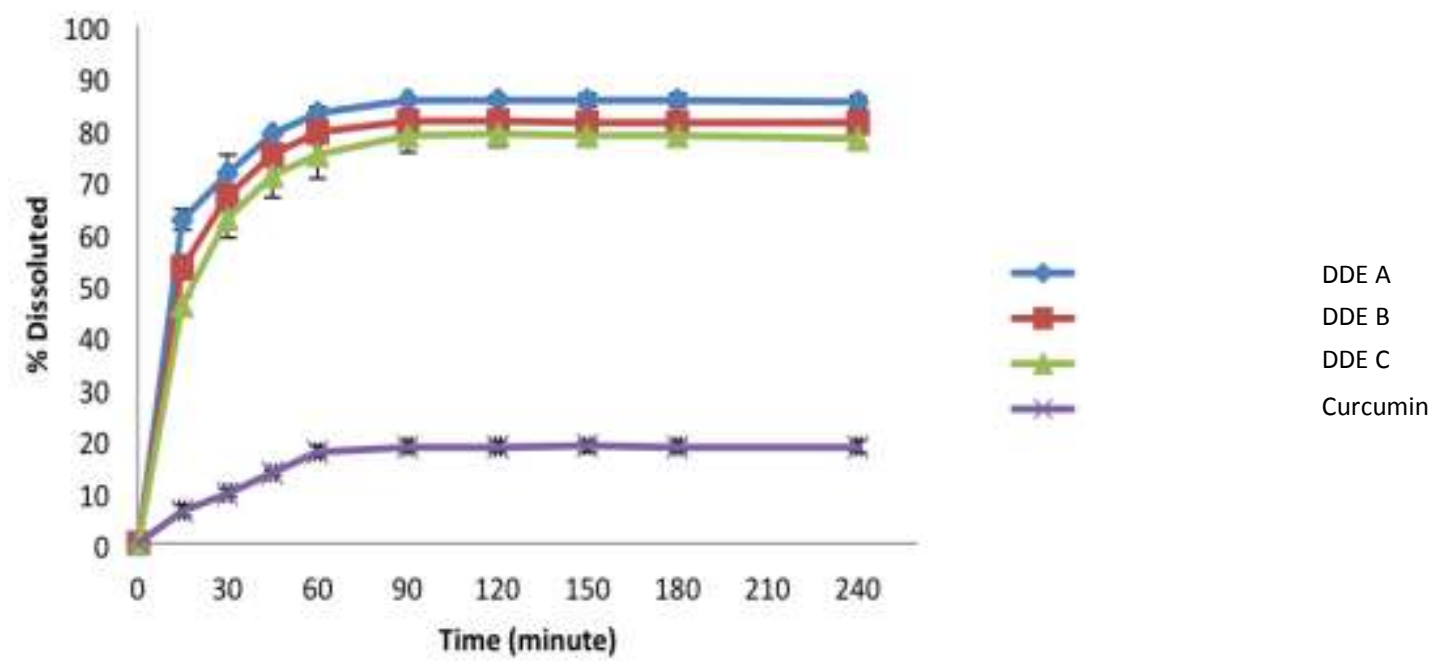

Fig. 4: Curcumin cumulative dissolution profiles of dry dispersible emulsion (DDE) and standard curcumin in medium phosphate buffer at $\mathrm{pH} 6.8$

Each point depicts the mean value $(n=3)$. Based on the results obtained the dissolution test showed that the percentage of dissolved solid lipid nanoparticle powder of formula $\mathrm{A}$ is greater than $\mathrm{B}$, while the method of dry dispersible emulsion powder in Formula B had higher dissolution percentage rate than formula C. An increase in the curcumin dissolution rate may be due to an increase in the solubility of dry dispersible emulsion. At the time of dry dispersible emulsion formation, curcumin is absorbed in the lipid then encapsulated by a water-soluble carrier. Also, particle size reduction can lead to increased surface area of the drug. Increased size can affect the solubility and dissolution rate of curcumin as a drug ingredient in the form of the dry dispersible emulsion. Other factors that influence the solubility is the change from a crystal structure to an amorphous form.

\section{Conclusion}

A dry dispersible emulsion containing curcumin can be formed using surfactants and lipids sodium caseinate and Virgin Coconut Oil along with high-speed homogenization techniques and ultrasonication. The dissolution rate of curcumin can be significantly improved with a dry dispersible emulsion formulation. In formula $\mathrm{A}$ and formula $\mathrm{C}$, the maximum dissolved curcumin increased by $83.65 \%, 81.53 \%$ in formula $\mathrm{B}$, and $79.12 \%$ in formula C. Meanwhile formula A which contained 7\% of sodium caseinate was the best formula as it produced particle sizes of $690.4 \mathrm{~nm}$ and a zeta potential value of $-29.77 \mathrm{mV}$. Formula B $(8 \%$ sodium caseinate) resulted in $941.3 \mathrm{~nm}$ particle size and a zeta potential value of $-21.79 \mathrm{mV}$. Meanwhile, formula C ( $9 \%$ sodium caseinate) led to a particle size of $1151 \mathrm{~nm}$ and a zeta potential value of $-27.51 \mathrm{mV}$.

\section{References}

1. Ajay Sav, Harita1Desai, Tarique Meer, Amin Purnima Solubility and Dissolution Rate Enhancement of Curcumin Using Kollidon VA64 by Solid Dispersion Technique, International Journal of PharmTech Research.2012,4(3) : 1055-1064

2. Franklyne Jonathan Sampath, Nadarajan Abinaya, Ebenazer Andrew, Tiwari Nisha, Mukherjee Amitava, Chandrasekaran Natrajan Preparation and Characterization of Edible Oil Nanoemulsion for Enhanced Stability and Oral Delivery of Curcumin, International Journal of Applied Pharmaceutics, 2018; 10 : 139-48, doi:10.22159/ijap.2018v10i6.28726.

3. Araujo, C.C. and Leon, L.L. Biological activities of Curcuma longa L., The Memórias do Instituto Oswaldo Cruz, 2001; 96: 723, doi:10.1590/S0074-02762001000500026.

4. Saipin, S., Sirima, M., Narubodde, P., Wiwat, P., \& Ruedeekorn, W. Development and evaluation of self-micro emulsifying liquid and pellet formulations of curcumin, and absorption studies in rats, 
European Journal of Pharmaceutics and Biopharmaceutics, 2010; 76: 475-85, doi:10.1016/j.ejpb.2010.07.011..

5. Humberstone, A.J. and William, N.C. Lipid-based vehicles for the oral delivery of poorly watersoluble drugs, Advanced Drug Delivery Reviews, 1997; 25: 103-28, doi:10.1016/S0169409X(96)00494-2.

6. Dollo, G., Le Corre, P., Guérin, A., Chevanne, F., Burgo, J.L. and Leverage, R. Spray-dried redispersible oil-in-water emulsion to improve oral bioavailability of poorly soluble drugs, European Journal of Pharmaceutical Sciences, 2003; 19: 273-80, doi:10.1016/S0928-0987(03)00134-9.

7. Mulik, R.S., Mönkkönen, J., Juvonen, R.O., Mahadik, K.R. and Paradkar, A.R.. Transferrin mediated solid lipid nanoparticles containing curcumin: enhanced in vitro anticancer activity by induction of apoptosis, International Journal of Pharmaceutics, 2010; 398: 190-203, doi:10.1016/j.ijpharm.2010.07.021.

8. Anant, R.P., Ambike, A.A., Jadhav, B.K. and Mahadik, K.R. Characterization of Curcumin - PVP solid dispersion obtained by spray drying. Int J Pharm 2003; 271: 281-86.

9. Tiyaboonchai, W., Tungpradit, W. and Pinyupa, P. Formulation and Characterization of Curcuminoids loaded Solid Lipid Nanoparticles, International Journal of Pharmaceutics, 2007; 337: 299-306.

10. $\mathrm{Yu}, \mathrm{H}$. and Huang, Q. Improving the oral bioavailability of curcumin using novel organogel-based nanoemulsions, Journal of Agricultural and Food Chemistry, 2012; 60:5373-79, doi:10.1021/jf300609p.

11. Jang, D.J., Kim,S. T., Oh, E.C. and Lee,K.Y. Enhanced oral bioavailability and antiasthmatic efficacy of curcumin using redispersible dry emulsion, Bio-Medical Materials and Engineering, 2014; 24: $917-$ 30 .

12. Eldem, T., Speiser, P. and Hincal, A. Optimization of Spray Dried and Congealed Lipid Microparticles and Characterization of Their Surface Morphology by SEM, Pharmaceutical Research, 1991; 8: 47-54, doi:10.1023/A:1015874121860.

13. Singh, P., Kumar, R., Sabapathy, S. N. and Bawa, A. S. Functional and edible uses of soy protein products,Comprehensive Reviews in Food Science and Food Safety, 2008; 7: 14-28, doi:10.1111/j.1541-4337.2007.00025.x.

14. Sharma, R.A., Gescher, A.J. and Steward, W.P.Curcumin: The story so far, European Journal of Cancer, 2005; 41 (13): 1959, doi:10.1016/j.ejca.2005.05.009.

15. Jayaprakasha, G. K., Rao, L. J. M., \& Sakariah, K. K. Antioxidant Activities of Curcumin, Demethoxycurcumin, Bisdemethoxycurcumin, Food Chemistry, 2005; 98(4): 720-24.

16. Frietas,C. and Muller, R.H. Correlation between long-term stability of solid lipid nanoparticles (SLN $\square$ ) and crystallinity of the lipid phase, European Journal of Pharmaceutics and Biopharmaceutics, 1999; 47: 125-32, doi:10.1016/S0939-6411(98)00074-5.

17. Sinko, P.J.,Allen Jr.,L.V., Popovich, N.G. andAnsel, H.C. Martin's Physical Pharmacy and Pharmaceutical Sciences. Philadelphia: Lippincott Williams \& Wilkins. 1996.

18. Li, L.C. and Tian, Y. Zeta Potential. In: Swarbrick, J., editor. Encyclopedia of Pharmaceutical Technology. (Ed. 1). New York: Marcel Dekker. 2007. 429-58.

19. Athira Geetha K Jyothi Alummoottil N Preparation and Characterization of Curcumin Loaded Cassava Starch Nanoparticles with improved cellular absorption, International Journal of Pharmacy and Pharmaceutical Sciences, 2014 ; 10: 171-76. 